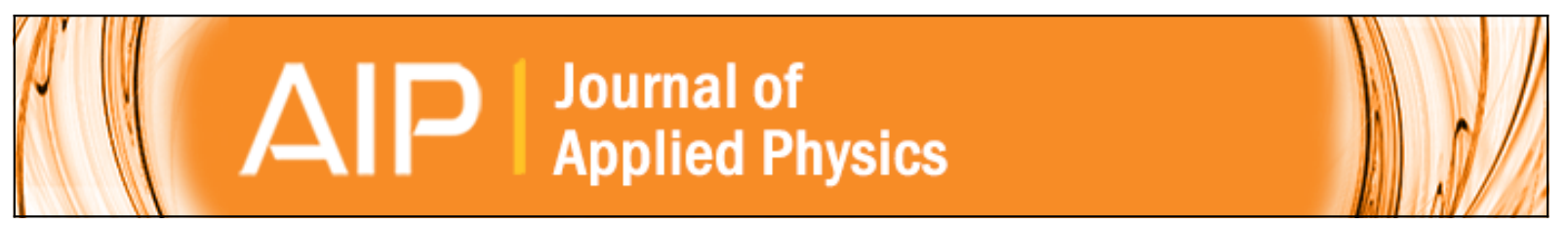

Evaluation of the two-photon absorption characteristics of GaSb/GaAs quantum rings

M. C. Wagener, P. J. Carrington, J. R. Botha, and A. Krier

Citation: Journal of Applied Physics 116, 044304 (2014); doi: 10.1063/1.4891223

View online: http://dx.doi.org/10.1063/1.4891223

View Table of Contents: http://scitation.aip.org/content/aip/journal/jap/116/4?ver=pdfcov

Published by the AIP Publishing

Articles you may be interested in

Photocapacitance study of type-II GaSb/GaAs quantum ring solar cells

J. Appl. Phys. 115, 014302 (2014); 10.1063/1.4861129

Simulation of the enhanced infrared photoresponse of type-II GaSb/GaAs quantum ring solar cells

Appl. Phys. Lett. 103, 063902 (2013); 10.1063/1.4818126

Enhanced infrared photo-response from $\mathrm{GaSb} / \mathrm{GaAs}$ quantum ring solar cells

Appl. Phys. Lett. 101, 231101 (2012); 10.1063/1.4768942

Thermal emission in type-II GaSb/GaAs quantum dots and prospects for intermediate band solar energy conversion

J. Appl. Phys. 111, 074514 (2012); 10.1063/1.3703467

Ultrafast nonlinear tuning of the reflection properties of AIGaAs photonic crystal waveguides by two-photon absorption

J. Appl. Phys. 96, 4729 (2004); 10.1063/1.1790569

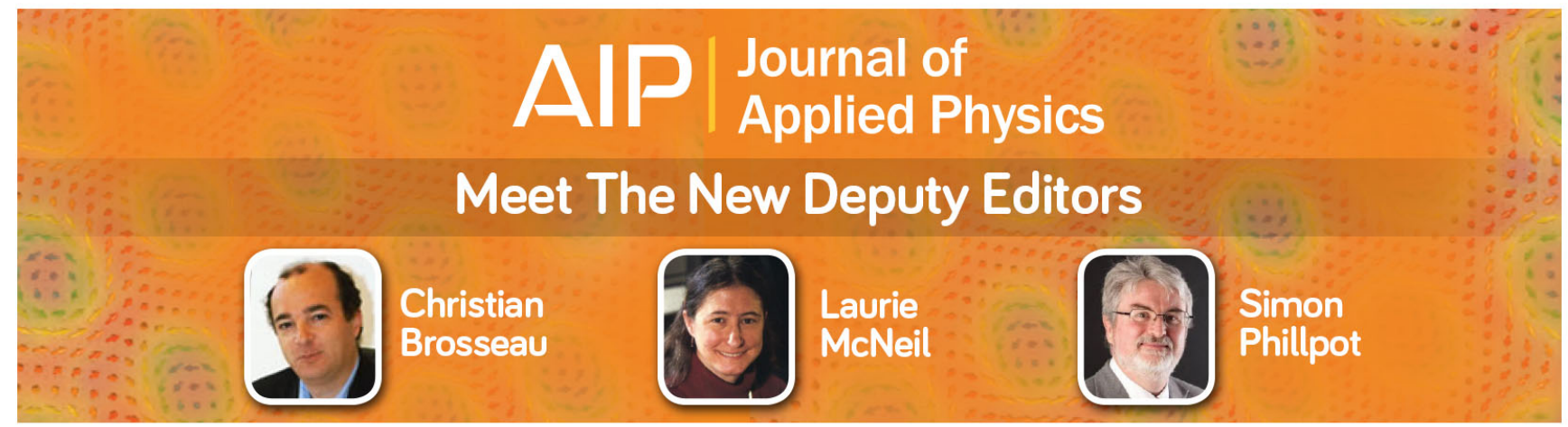




\title{
Evaluation of the two-photon absorption characteristics of GaSb/GaAs quantum rings
}

\author{
M. C. Wagener, ${ }^{1}$ P. J. Carrington, ${ }^{2}$ J. R. Botha, ${ }^{1}$ and A. $\mathrm{Krier}^{3}$ \\ ${ }^{1}$ Department of Physics, Nelson Mandela Metropolitan University, Port Elizabeth 6031, South Africa \\ ${ }^{2}$ Department of Electronic and Electrical Engineering, University College London, London, United Kingdom \\ ${ }^{3}$ Department of Physics, Lancaster University, Lancaster LA1 4YB, United Kingdom
}

(Received 17 April 2014; accepted 13 July 2014; published online 23 July 2014)

\begin{abstract}
The optical parameters describing the sub-bandgap response of $\mathrm{GaSb} / \mathrm{GaAs}$ quantum rings solar cells have been obtained from photocurrent measurements using a modulated pseudomonochromatic light source in combination with a second, continuous photo-filling source. By controlling the charge state of the quantum rings, the photoemission cross-sections describing the two-photon sub-bandgap transitions could be determined independently. Temperature dependent photo-response measurements also revealed that the barrier for thermal hole emission from the quantum rings is significantly below the quantum ring localisation energy. The temperature dependence of the sub-bandgap photo-response of the solar cell is also described in terms of the photo- and thermal-emission characteristics of the quantum rings. (C) 2014 AIP Publishing LLC.

[http://dx.doi.org/10.1063/1.4891223]
\end{abstract}

\section{INTRODUCTION}

Since the conversion efficiency of solar energy by a single junction solar cell is ultimately limited by the loss of sub-bandgap photons, ${ }^{1}$ a variety of so called third generation solar cells have been proposed to try and surpass the singlejunction limit. ${ }^{2}$ An interesting approach is the use of an intermediate-band (IB) within the forbidden gap of a single junction cell, ${ }^{3}$ with the IB used to generate electron-hole pairs through a two-photon process. This IB concept is illustrated in Fig. 1(a): when two photons with energy below the bandgap energy are absorbed, the photocurrent results from a photon exciting an electron from the IB to the conduction band (CB), and the photoemission of a hole from the IB to the valence band. The absorption strength of the various transitions is denoted by their respective optical crosssections, such as valence- to intermediate-band absorption $\left(\sigma_{\mathrm{VI}}\right)$, intermediate- to conduction band absorption $\left(\sigma_{\mathrm{IC}}\right)$, and direct absorption across the forbidden gap $\left(\sigma_{\mathrm{VC}}\right)$. Alongside the optical processes are the various thermal and Auger transitions of which the thermal emission of holes from the intermediate-band to the valence band $\left(e_{\mathrm{h}}\right)$ is the most relevant for this study. Since the IB concept heavily relies on a predominance of optical generation, controlling the various IB processes is of great importance.

Although the desired sub-bandgap response of IB solar cells have been achieved using various strategies, such as the use of dilute semiconductor alloys ${ }^{4}$ and low-dimensional quantum structures, ${ }^{5}$ a common short-coming has been a detrimental decrease in the open-circuit voltage (VOC) of the solar cell. ${ }^{6,7}$ The reason for the reduced VOC is believed to be due to thermal escape of carriers from the IB dominating over two-photon absorption, leading to the output voltage ultimately being limited by the sub-bandgap energy $E_{\mathrm{IB}} \cdot{ }^{3} \mathrm{~A}$ novel approach to the IB cell has been the incorporation of $\mathrm{GaSb} /$ GaAs quantum rings (QRs) into the active region of a $\mathrm{p}-\mathrm{i}-\mathrm{n}$ structure. ${ }^{6}$ Due to the characteristic type-II band alignment of

the $\mathrm{GaSb} / \mathrm{GaAs}$ heterojunction, holes are strongly confined within the GaSb nanostructure, whereas electrons are weakly bound on the perimeter of the QR. Figure 1(b) depicts the type-II band alignment of the GaSb quantum rings. As a results of a relatively small effective mass, ${ }^{8}$ the localization of holes within the GaSb ring results in a discrete hole state (dashed line in Fig. 1(b)). The inhomogeneous size distribution of the quantum rings, however, gives rise to a band of holes states roughly $400 \mathrm{meV}$ above the GaAs valence band (VB). ${ }^{9}$ This band of localized hole states has previously been shown to extend the photo-response well below the GaAs absorption edge. ${ }^{6,9}$ As with other IB solar cells, the increased photocurrent is accompanied by a significant decrease in the VOC of the solar cell. ${ }^{6}$ A recent approach has been the use of blocking layers to hinder the current path between the IB and the $\mathrm{CB}$ of the junction material. ${ }^{10}$ Although a significant recovery of the VOC was demonstrated, the photocurrent was drastically impeded by the blocking layers. At present, the only meaningful recovery of the VOC is by reducing the operating temperatures of the solar cell or by using concentrated

(a)

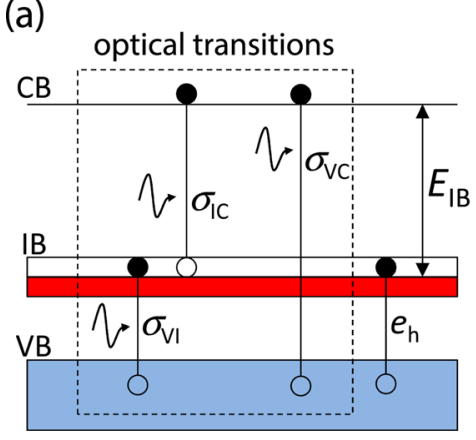

FIG. 1. Illustration of the optical and thermal transitions of an intermediateband solar cell (a), with sub-bandgap energy $E_{\mathrm{IB}}$. The type-II band structure of the $\mathrm{GaSb} / \mathrm{GaAs}$ heterojunction (b) results in hole confinement, with the charging and discharging of the quantum structures governed by electron emission $\left(\sigma_{\mathrm{IC}}\right)$ and optical/thermal hole emission $\left(\sigma_{\mathrm{VI}}, e_{\mathrm{h}}\right)$, respectively. 
excitation. ${ }^{11}$ It is therefore apparent that any future progress will require knowledge of the optical and the capture/emission kinetics of the quantum structures. The authors have therefore set out to determine the optical properties of the intermediateband of quantum ring states, specifically the photoemission cross-sections associated with electron and hole emission from the rings (see Fig. 1).

\section{EXPERIMENTAL DETAILS}

The solar cell structures used in this study were deposited using a VG-V80H molecular beam epitaxy reactor. A detailed description of the growth procedure and the device structure has been presented elsewhere. ${ }^{6}$ The active region of the p-i-n diode contains ten $\mathrm{GaSb} / \mathrm{GaAs} \mathrm{QR}$ stacks, each with a typical surface density of $10^{10}$ rings $/ \mathrm{cm}^{2}$. Crosssectional transmission electron microscopy images showed the rings to have an outer and inner diameter of roughly $23 \mathrm{~nm}$ and $10 \mathrm{~nm}$, respectively, with a height of approximately $1.7 \mathrm{~nm}$. $^{6}$ These structures were processed into circular solar cells with a diameter of $1 \mathrm{~mm}$ using standard photolithography. The setup used for the photo-response measurements is shown in Fig. 2. Both a modulated primary light source and a second, continuous source were used. For the primary light source, a $100 \mathrm{~W}$ tungsten halogen lamp, in conjunction with a monochromator (equipped with a 600 lines/ $\mathrm{mm}$ ruled diffraction grating) and an achromatic depolarizer was used, whereas a $7 \mathrm{~mW}, 525 \mathrm{~nm}$ light emitting diode (LED) was introduced as the second source. The optical chopper was placed at the entrance slit of the monochromator in order to minimize any reflections from the second source off the chopper wheel. The spectral response of the solar cell was obtained by measuring the short-circuit photocurrent using a Keithley 6487 pico-ammeter and a phasesensitive lock-in amplifier. Appropriate long-pass filters were inserted to remove higher order diffraction lines. The temperature dependent photo-response measurements were performed using a close-cycle helium cryostat, with the solar cell surface oriented normal to the primary optical axis.

\section{RESULTS AND DISCUSSION}

In a previous study, ${ }^{12}$ photocapacitance measurements revealed that the $\mathrm{GaSb} / \mathrm{GaAs}$ quantum rings could be optically charged or optically discharged, depending on the excitation used, with photon energies above and below $0.9 \mathrm{eV}$

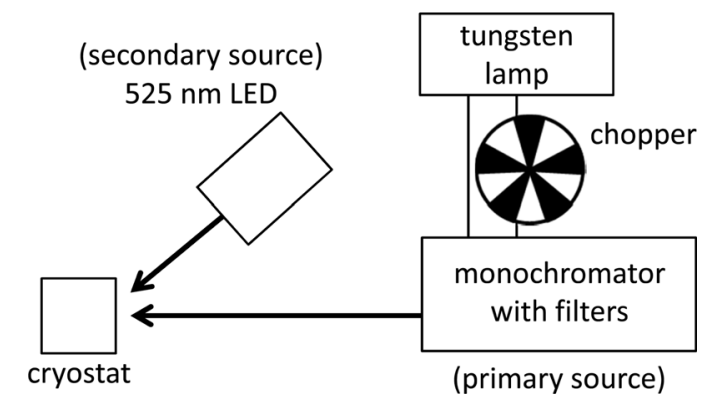

FIG. 2. Description of the photo-response setup used. The solar cell was illuminated using a modulated monochromatic source (primary), in combination with a continuous, supra-bandgap photon source (secondary). leading to charging and discharging, respectively. In the case of the spectral response of the solar cells, the quantum ring contribution to the photocurrent was also found to decrease sharply for excitation energies below roughly $1 \mathrm{eV}$. Since the QR states were shown to be distributed near the valence band maximum (VBM) ${ }^{12}$ the main contribution to the photocurrent appears to be due to optical transitions between the QR hole states and the conduction band, with no significant photoemission of holes to the valence band. A likely explanation for the absence of transitions to the valence band emerges when considering the charge state of the rings during optical excitation. For photon energies above the subbandgap energy, $E_{\mathrm{IB}}$, the photoemission of electrons to the conduction band will be accompanied by the thermal (and optical) emission of localized holes to the valence band. However, for photon energies below the sub-bandgap energy, hole emission will dominate, leading to the complete discharging of the quantum rings. Optical excitation with the primary source alone will therefore result in the hole and electron contributions decreasing concurrently for photon energies below $E_{\mathrm{IB}}$; whereas if a steady QR charge could be maintained, the primary source could potentially contribute for photon energies below $E_{\mathrm{IB}}$ through the optical emission of holes to the valence band.

In order to maintain the QR charge, the solar cell was therefore illuminated with an additional, above bandgap excitation source. Some of the excess holes generated by the second source are then trapped within the quantum rings, leading to a steady-state quantum ring charge governed by the thermal and optical emission kinetics of the rings. The alternative setup used for the photo-response measurements therefore consists of a modulated pseudo-monochromatic (primary) light source in combination with a continuous (secondary) $525 \mathrm{~nm}$ source. In order to maximize the hole density within the quantum rings, the temperature was also decreased for the photo-response measurements. The $16 \mathrm{~K}$ photocurrent spectra of the $\mathrm{GaSb} / \mathrm{GaAs}$ solar cell measured with and without the second excitation source is compared in Fig. 3(a). It is clear that the second source significantly increases the response to photon energies below $1.1 \mathrm{eV}$, with only a slight change in the spectral response at higher photon energies. Since the second excitation source is expected to maintain the QR charge through the trapping of photogenerated holes, the authors attribute the increased response to the photoemission of holes from the rings. Note that since only modulated signals contributes to the phase-sensitive measurement, the enhanced photo-response observed below $1.1 \mathrm{eV}$ is due to an increased contribution by the primary light source, with the second light source only impacting the charge state of the quantum rings. It should also be noted that the noise level of the photocurrent measurements increased only slightly (from $2 \times 10^{-11}$ to $5 \times 10^{-11} \mathrm{~A} / \mathrm{cm}^{2}$ ) due to some scattering of the second source off the chopper blades. The increased photocurrent for excitation energies below $1.1 \mathrm{eV}$ is therefore clearly not attributed to any modulation of the second source. The position of the band of QR states relative to the valence band maximum also suggests that the entire band would contribute for photon energies above $0.5 \mathrm{eV}$, thereby explaining the relatively constant 

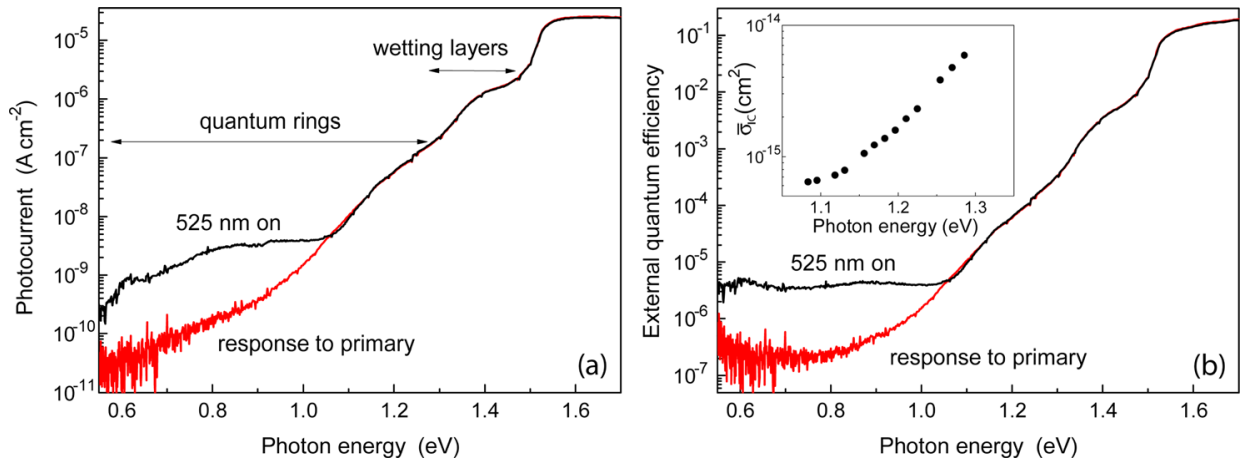

FIG. 3. Low temperature (16 K) photocurrent spectra (a) of the $\mathrm{GaSb} / \mathrm{GaAs}$ quantum rings and wetting layers obtained using a modulated pseudomonochromatic light source (primary) with and without a second continuous $525 \mathrm{~nm}$ source. The external quantum efficiency obtained with the two excitation conditions are compared in (b). The inset gives the spectral dependence of the electron photoemission cross-section from the intermediateband. response in Fig. 3(b) at low photon energies. Substituting the $525 \mathrm{~nm}$ source with the broad spectrum of a tungstenhalogen lamp produced essentially the same result (not shown).

In the case of an inhomogeneous size distribution of quantum rings, we can assume that hole confinement results in a continuous band of states, with the quantum efficiency of the ensemble of VB to IB transitions, $Q E_{\mathrm{VI}}$, (or the IB to $\mathrm{CB}$ transitions, $Q E_{\mathrm{IC}}$ ) for the particular photon energy $E_{P}$, given by the following relations:

$$
\begin{array}{r}
Q E_{V I}\left(E_{P}\right)=\int_{0}^{E_{P}} \sigma_{V I} f N(E) d E=\bar{\sigma}_{V I} \int_{0}^{E_{P}} f N(E) d E \\
Q E_{I C}\left(E_{P}\right)=\int_{E_{g}-E_{P}}^{E_{g}} \sigma_{I C}(1-f) N(E) d E \\
=\bar{\sigma}_{I C} \int_{E_{g}-E_{P}}^{E_{g}}(1-f) N(E) d E
\end{array}
$$

where $\sigma_{\mathrm{VI}}$ and $\sigma_{\mathrm{IC}}$ represent the two intermediate-band related photoemission cross-sections described in Fig. 1(a), $N(E)$ the energy distribution of the QR density of states relative to the VBM (with $E=0$ at the VBM), $f$ the fraction of QR states occupied and $E_{\mathrm{g}}$ the GaAs bandgap energy. The mean photoemission cross-sections associated with electron and hole emission from the intermediate-band are denoted by $\bar{\sigma}_{I C}\left(E_{P}\right)$ and $\bar{\sigma}_{V I}\left(E_{P}\right)$, respectively. The band of QR hole states was previously shown to be centered $407 \mathrm{meV}$ above the VBM, ${ }^{12}$ with an integrated state density of $4 \times 10^{10} \mathrm{~cm}^{-2}$. Substituting the integral in Eq. (1), the quantum efficiency (photocurrent divided by the photon flux density) estimated for the VB to IB transition of $Q E_{\mathrm{VI}} \approx 4 \times 10^{-6}$ (Fig. 3(b)) relates to a mean hole photoemission cross-section of $\bar{\sigma}_{V I} \approx 10^{-16} \mathrm{~cm}^{2}$. In the case of the IB to CB transitions, however, the segment of the IB (integral in Eq. (2)) needs to be known. Since the energy distribution of the QR charge has previously been described in detail in Ref. 12, a mean photoemission cross-section of $\bar{\sigma}_{I C} \approx 7 \times 10^{-16} \mathrm{~cm}^{2}$ could be estimated for transitions near the $\Gamma$-point $\left(E_{\mathrm{P}}=1.1 \mathrm{eV}\right)$, increasing to $7 \times 10^{-15} \mathrm{~cm}^{2}$ for $E_{\mathrm{P}}=1.3 \mathrm{eV}$ (see inset in Fig. 3(b)). Note that since the IB represents the localized hole states associated with a stack of quantum rings, the cross-section of a single $\mathrm{QR}$ layer would be a factor 10 smaller.

Analogous to the enhanced photocurrent observed for the interband transitions would be the absorption spectra obtained from photoinduced infrared absorption spectroscopy. ${ }^{13}$
Although the photoluminescence (PL) of type-II heterostructures have been intensively studied, very few absorption results have been reported. In the case of a type-I band alignment, Sauvage et al. estimated the optical cross-sections of the in-plane polarized absorption of self-assembled InAs/ GaAs quantum dots to be approximately $1.8 \times 10^{-16} \mathrm{~cm}^{2}$ and $1.9 \times 10^{-16} \mathrm{~cm}^{2}$ for electron and hole emission, respectively. ${ }^{13}$ However, in order to relate the optical cross-section of the InAs quantum dots to the absorption spectra, the density of occupied states had to be estimated using a doped reference sample. By combining the photocurrent and photocapacitance characteristics of the solar cell, we therefore have the clear advantage of relating the absorption strength directly to the density of QR states.

Returning to Eq. (1), it is apparent that irrespective of the occupation of states, $Q E_{\mathrm{VI}}$ starts to exceed $Q E_{\mathrm{IC}}$ as the integrated density of states in Eq. (2) becomes negligible, i.e., when the photon energy is below the sub-bandgap energy of the quantum rings, i.e., $E_{\mathrm{P}}<E_{\mathrm{IB}}$. The difference in quantum efficiency of the two spectra is depicted in Fig. 4(a). The increased contribution by $Q E_{\mathrm{VI}}$ is illustrated by the increase in the relative quantum efficiency within the grey segment. Since $Q E_{\mathrm{VI}}$ is expected to be relatively constant, the band of QR states is expected to roughly lie between $E_{\mathrm{g}}-1.09 \mathrm{eV}=430 \mathrm{meV}$ and $E_{\mathrm{g}}-0.87 \mathrm{eV}=650 \mathrm{meV}$ above the GaAs VBM, with the relative increase in the quantum efficiency at low photon energies described by the right-hand integral in Eq. (2). Treating the optical cross-section as a variable, the density of states $N(E)$ is then related to the partial derivative of the relative $\mathrm{QE}$ in terms of the photon energy. Figure 4(b) depicts the energy dependence of the QR state density obtained using the delta spectrum in Figure 4(a). By adjusting the energy axis relative to the GaAs bandgap (i.e., absciss $a=E_{\mathrm{g}}-E_{\mathrm{P}}$ ), we find that the $\mathrm{QR}$ state density has a distribution centered $409 \mathrm{meV}$ above the VBM, with the distributions well matched when using a constant photoemission cross-section of $\bar{\sigma}_{I C}=3 \times 10^{-16} \mathrm{~cm}^{2}$ for photon energies below $1.1 \mathrm{eV}$.

In an earlier study, the density of localized hole states was estimated from the excitation dependence of the photocapacitance, with the energy distribution shown to be composed of two Gaussian distributions centered $188 \mathrm{meV}$ and $407 \mathrm{meV}$ above the VBM (solid line in Fig. 4(b)). ${ }^{12}$ Based on the PL spectra of similar structures, the two bands have been ascribed to the wetting layers (WLs) and the quantum rings, respectively. ${ }^{14}$ The distribution obtained from the 

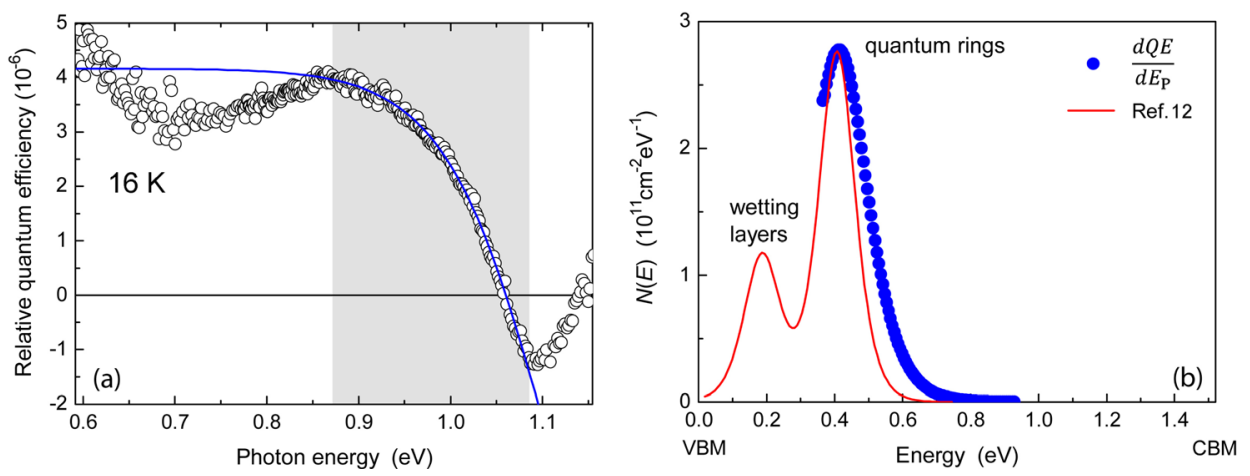

FIG. 4. Relative quantum efficiency (a) obtained by subtracting the efficiency spectra in Fig. 3(b) from one-another. The solid line represents a fit of the relative QE using a logistic function. The density of hole states (b) was obtained by differentiating the relative QE within the grey segment in (a). The energy axis has been adjusted relative to the GaAs bandgap (i.e., bandgap energy minus transition energy). The solid line in (b) represents the density of states obtained by photocapacitance spectroscopy performed on the same structure. ${ }^{12}$ The two approaches were best related when assuming a constant photoemission crosssection of $\bar{\sigma}_{I C}=3 \times 10^{-16} \mathrm{~cm}^{2}$ for $E_{P}<1.1 \mathrm{eV}$.

enhanced QE spectrum is found to be well matched to the photocapacitance analysis, with the slight broadening attributed to the sensitivity of the distribution to the differential operation. Although the enhanced response depicted in Fig. 3 (b) is clearly related to the energy distribution expected for the QR states, the absence of any enhanced WL response suggests that thermal hole emission (or tunneling) from the WL states to the GaAs valence band dominates over photoemission.

An important observation related to the addition of a second excitation source during the photo-response measurements is the slight decrease in the $\mathrm{QE}$ for photon energies above $1.06 \mathrm{eV}$ (see Fig. 3(b)). This is depicted more clearly by the negative relative response in Fig. 4(a) for photon energies above $1.06 \mathrm{eV}$. This decrease in the QR response was also found to be proportional to the intensity of the second excitation source (not shown). Although a decrease in $Q E_{\mathrm{IC}}$ is most likely attributed to an increase in the number of oсcupied hole states (increase in total QR charge due to hole trapping), it appears that the gain in $Q E_{\mathrm{VI}}$ significantly exceeds the overall loss in $Q E_{\mathrm{IC}}$. A possible explanation for the relative insensitivity of $Q E_{\mathrm{IC}}$ to the occupation state of the quantum rings could be two-photon excitation, whereby a combination of photo-filling (IB to CB transitions) and emptying (VB to IB transitions) of the $\mathrm{QR}$ states results in the photo-generation of electron-hole pairs. A detailed balance model for the IB solar cell was developed by Strandberg and Reenaas ${ }^{15}$ to describe the photo-filling of the IB under different levels of light concentration. In the case of low light intensities, the need to prefill the IB by means of doping was emphasized, whereas photo-filling was found to be sufficient under several hundred suns of concentrated light. Although two-photon absorption is typically described for a continuous, partially filled band of intermediate states, the QR hole states are anticipated to be spatially isolated from each other. Based on the band offset and effective hole mass of the GaSb/GaAs quantum rings, ${ }^{9}$ the evanescent decay within the GaAs barrier should have an attenuation factor in the order of $10^{3} \mathrm{~nm}^{-1}$ for the observed distribution of QR states. Due to the $40 \mathrm{~nm}$ thick barrier layer between successive QR stacks, the IB can therefore be thought of as an ensemble of discrete, spatially isolated hole states. The charge state of a particular QR during illumination can then be considered to be independent of the remaining distribution of $\mathrm{QR}$ states, whether charged or not. The two-photon absorption associated with the band of QR states is then instead represented by an effective optical cross-section $\bar{\sigma}_{V I C}=\frac{1}{2}\left(\bar{\sigma}_{V I} \bar{\sigma}_{I C}\right) /\left(\bar{\sigma}_{V I}+\bar{\sigma}_{I C}\right)$. In other words, for sufficiently low temperatures (i.e., when thermal hole emission is negligible), the spectral dependence of the optical generation rate $e^{o}$ would be described by

$$
\begin{gathered}
e^{o}\left(E_{P}\right)=\frac{e_{I C}^{o} e_{V I}^{o}}{e_{I C}^{o}+e_{V I}^{o}}, \\
e_{V I}^{o}\left(E_{P}\right)=\frac{1}{2} \Phi\left(E_{P}\right) \int_{E_{g}-E_{P}}^{E_{P}} \sigma_{V I} N(E) d E, \\
e_{I C}^{o}\left(E_{P}\right)=\frac{1}{2} \Phi\left(E_{P}\right) \int_{E_{g}-E_{P}}^{E_{g}} \sigma_{I C} N(E) d E,
\end{gathered}
$$

where $\Phi\left(E_{P}\right)$ represents the energy distribution of the photon flux density. Note that the occupation factor $f$ has been set to $50 \%$ in Eqs. (4) and (5), with the contribution by the two integrals assumed to be roughly equivalent for the band of QR states. In the absence of a second charging source, a photon with energy above the sub-bandgap energy $\left(E_{\mathrm{IB}}\right.$ $\approx 1.1 \mathrm{eV}$ at $16 \mathrm{~K}$ ) would be required for every VB to IB transition. The two-photon absorption edge associated with the effective emission rate described in Eq. (3) will therefore still be determined by the sub-bandgap energy $E_{\mathrm{IB}}$ of the intermediate-band.

In order to describe the thermal hole emission kinetics of the QR states, the relative quantum efficiency presented in Fig. 4(a) was measured for a range of temperatures. Figure 5(a) depicts the temperature dependence of the VB to IB transitions. The photoemission of holes from the IB to the valence band is found to gradually decrease above $50 \mathrm{~K}$, with no discernible optical hole emission above $200 \mathrm{~K}$. Two-photon excitation via the localized QR states is therefore expected to dominate the sub-bandgap response at low temperatures, with electron photoemission and thermal hole emission governing the photo-response above $200 \mathrm{~K}$. The temperature 

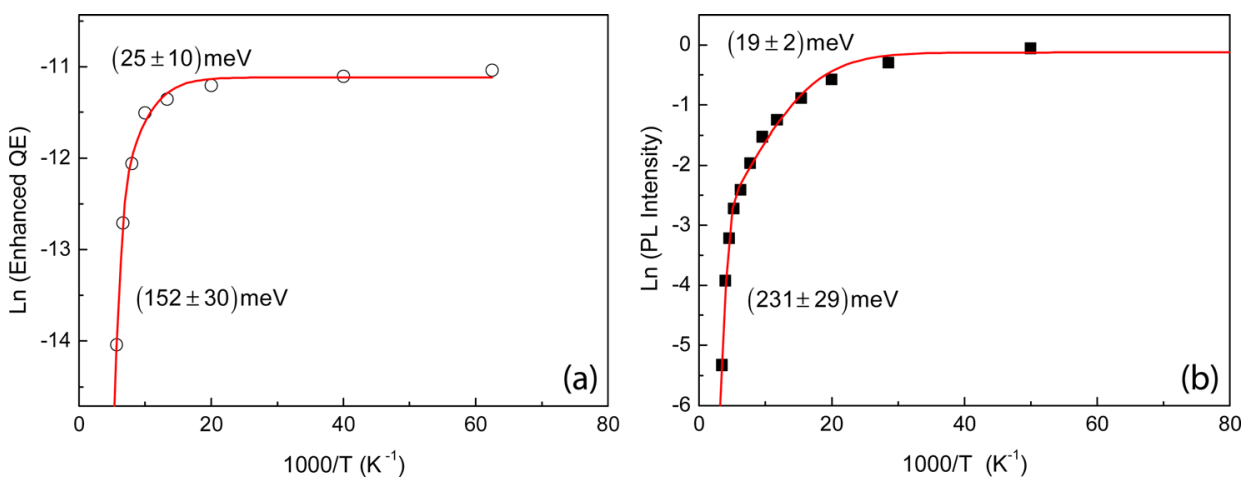

FIG. 5. Temperature dependence of (a) the VB to IB contribution to the quantum efficiency of the solar cell and (b) the integrated QR photoluminescence of the solar cell. The photoluminescence was obtained using an unprocessed device structure. The solid lines and the respective energies represent the fitting results obtained using Eq. (6). dependence of $Q E_{V I}$ is well described across the whole temperature range by the following dual loss equation:

$$
I(T)=\frac{I_{0}}{1+A_{1} \exp \left(-E_{1} / k T\right)+A_{2} \exp \left(-E_{2} / k T\right)},
$$

where $I_{0}$ represents the zero Kelvin response, $k$ is the Boltzmann constant, and $E_{1}$ and $E_{2}$ are the activation energies of the quenching processes. From the best fit of Eq. (6), we obtain an activation energy of $(152 \pm 30) \mathrm{meV}$ for the thermal quenching of the hole current at high temperatures. This compares reasonably well with the thermal quenching observed for the QR photoluminescence, with the intensity (Fig. 5(b)) exhibiting an activation energy of $(231 \pm 29)$ $\mathrm{meV}$. As expected, the $\mathrm{QR}$ photoluminescence is closely related to the hole emission kinetics of the quantum rings. Furthermore, the $219 \mathrm{meV}$ energy difference between the mean hole localization energies of the quantum rings and the wetting layers (Fig. 4(b)) suggests that thermal hole emission from the quantum rings occur via the wetting layer, leading to a notably reduced emission barrier.

Even though hole emission from the quantum rings is found to be highly temperature dependent, decreasing by two orders of magnitude between $50 \mathrm{~K}$ and $200 \mathrm{~K}$, the total sub-bandgap response of the solar cell is found to exhibit a significantly weaker temperature dependence. Figure 6 depicts the $1.1 \mathrm{eV}$ photo-response of the solar cell measured between $16 \mathrm{~K}$ and $300 \mathrm{~K}$ using the primary light source alone. In order to describe the temperature dependence of the sub-bandgap response, we relate the photocurrent $J_{P C}$ to the optical generation rates (i.e., $e_{V I}^{o}$ and $e_{I C}^{o}$ ), as well as the thermal hole emission rate $e_{h}$, using the relation

$$
J_{P C}\left(E_{P}\right)=\beta q \frac{e_{I C}^{o}\left(e_{V I}^{o}+e_{h}\right)}{e_{I C}^{o}+e_{V I}^{o}+e_{h}}
$$

where $\beta$ denotes the carrier extraction efficiency of the solar cell and $q$ the unit charge. Since the QR stacks are distributed within the space-charge region of the junction, we can assume $\beta$ to be constant and near unity throughout the $\mathrm{QR}$ distribution. We have also assumed that the sub-bandgap reflectivity is negligible for the spectral range considered. For sufficiently high temperatures, where $e_{h} \gg e^{o}$ (i.e., thermal emission greatly exceeds the optical transition rates), the photocurrent is anticipated to be proportional to $e_{I C}^{o}$ (i.e., the optical IB to CB transition rate), whereas the photocurrent will be determined by the effective photoemission rate $e^{o}$ (Eq. (3)) at sufficiently low temperatures (i.e., $e_{h} \ll e_{V I}^{o}$ ). The quantum efficiency associated with the band of QR states is therefore expected to increase by a factor $\left(1+\bar{\sigma}_{I C} / \bar{\sigma}_{V I}\right)$ with temperature, displaying two clear temperature regimes.

Figure 6 gives the temperature dependence of the QR related response for photon energies lying within $1.10 \pm 0.05 \mathrm{eV}$. Due to the temperature dependence of the GaAs bandgap, the spectral response curves were translated in order to match the GaAs absorption edge of each of the spectra (not shown). From Eq. (7), the ratio between the high and low temperature values relates to a photoemission crosssection ratio of $\bar{\sigma}_{I C} / \bar{\sigma}_{V I} \approx 3$. The spectral dependence of the QR response was previously simulated by describing the optical cross-section using Lucovsky's $\delta$-potential model. ${ }^{9}$ Based on the relative effective masses of the GaSb/GaAs heterostructure, ${ }^{8}$ as well as the position of the QR states within the gap (i.e., $407 \mathrm{meV}$ above the VBM), a ratio of $\bar{\sigma}_{I C} / \bar{\sigma}_{V I} \sim 0.5$ would be expected for deep level optical transitions near the $\Gamma$-point. The optical cross-sections describing the intermediate-band transitions therefore appear to be better matched than initially deduced from Fig. 3(b), with the small value of $\bar{\sigma}_{V I} \approx 10^{-16} \mathrm{~cm}^{2}$ likely due to the incomplete filling of the $\mathrm{QR}$ states during excitation with the second source (i.e., $f<1$ in Eqs. (1) and (2)). The enhanced

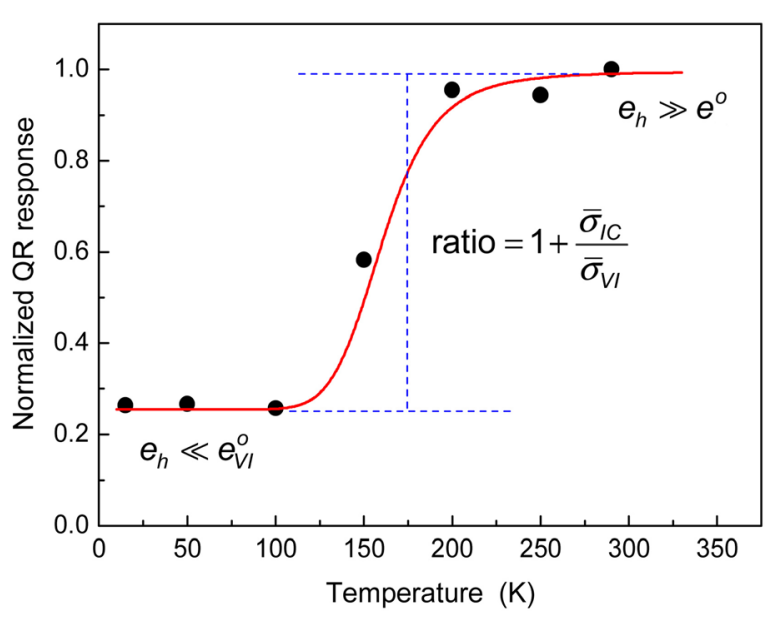

FIG. 6. Temperature dependence of the external quantum efficiency of the $\mathrm{GaSb} / \mathrm{GaAs}$ quantum rings for $E_{P} \sim 1.1 \mathrm{eV}$. The solid line represents the best fit obtained using Eq. (7) and an activation energy of $152 \mathrm{meV}$ (obtained from Fig. 5(a)) for the thermal emission of holes from the quantum rings. Note the linear scale for the ordinate axis. The ratio between the high and low temperature responses relates to $\bar{\sigma}_{I C} / \bar{\sigma}_{V I} \approx 3$ for IB to $\mathrm{CB}$ transitions near the $\Gamma$-point. 
TABLE I. Summary of the optical and electrical parameters obtained for the GaSb/GaAs quantum rings. Similar device structures were used for all of the results, with the photoemission cross-sections and density of hole states representative of a solar cell with 10 imbedded quantum ring layers.

\begin{tabular}{lccc}
\hline \hline Parameters & This study & Photocapacitance Spectroscopy $^{12}$ & Photocurrent Spectroscopy $^{9}$ \\
\hline Electron photoemission cross-section near $\Gamma$-point, $\bar{\sigma}_{I C}\left(E_{P}=1.1 \mathrm{eV}\right)$ & $7 \times 10^{-16} \mathrm{~cm}^{2}$ & $\ldots$ & $\ldots$ \\
Hole photoemission cross-section $\bar{\sigma}_{V I}\left(E_{P}<1.1 \mathrm{eV}\right)$ & $10^{-16} \mathrm{~cm}^{2}$ & $\ldots$ & $\ldots$ \\
Photoemission cross-section ratio $\bar{\sigma}_{I C} / \bar{\sigma}_{V I}\left(E_{P}=1.1 \mathrm{eV}\right)$ & $2.5 \pm 0.5$ & $\ldots$ & $\ldots$ \\
Thermal hole emission barrier & $(152 \pm 30) \mathrm{meV}$ & $\ldots$ & $\ldots$ \\
Mean QR localisation energy & $409 \mathrm{meV}$ & $407 \mathrm{meV}^{-2}$ & $\ldots 380 \mathrm{meV}$ \\
Density of quantum ring states & $\ldots$ & $4 \times 10^{10} \mathrm{~cm}^{-2}$ & $\ldots$ \\
\hline \hline
\end{tabular}

photo-response $\left(Q E_{\mathrm{VI}}\right)$ observed can therefore potentially match the primary response $\left(Q E_{\mathrm{IC}}\right)$ if a sufficient $\mathrm{QR}$ charge could be maintained, e.g., through doping or increased photo-filling. Table I lists the various parameters used to describe the short-circuit photo-response of the GaSb/GaAs solar cells. By assuming that the optical excitation between the localized hole states and the conduction band extends beyond the $\Gamma(k=0)$ point, the energy distribution of the QR states could be consistently described by each of the three approaches considered.

\section{CONCLUSIONS}

By relating the quantum efficiency of the quantum ring photo-response to the integrated density of hole states, a mean optical cross-section of $7 \times 10^{-16} \mathrm{~cm}^{2}$ could be estimated for photoemission of electrons from the band of quantum ring states to the conduction band minimum. Using a second excitation source to maintain the quantum ring charge, optical hole emission was observed at low temperatures, confirming the two-photon contribution to the $\mathrm{GaSb}$ / GaAs solar cell response. The optical emission of holes to the valence band has also been shown to exceed thermal emission for temperature below $200 \mathrm{~K}$, with the hole emission rate exhibiting a thermal activation energy of $(152 \pm 30) \mathrm{meV}$. Based on the average localisation energy of holes within the quantum rings, as well as the relative position of the wetting layer states, the authors propose that the effective hole emission barrier is significantly reduced by the proximity of the wetting layer. The temperature dependence of the sub-bandgap photo-response has also been described in terms of the optical and thermal emission rates, with the weak temperature dependence exhibited ascribed to closely matched electron and hole photoemission cross-sections.

\section{ACKNOWLEDGMENTS}

Financial support for this work was provided from EPSRC (Grant No. EP/G070334/1), as well as by the South African Research Chairs Initiative of the Department of Science and Technology, South African National Research Foundation, and NMMU. P.J.C. acknowledges support from the Royal Academy of Engineering through a Research Fellowship.

${ }^{1}$ W. Shockley and H. J. Queisser, J. Appl. Phys. 32, 510 (1961).

${ }^{2}$ M. A. Green, Third Generation Photovoltaics (Springer-Verlag, Berlin, 2003).

${ }^{3}$ A. Martí, E. Antolín, C. R. Stanley, C. D. Farmer, N. López, P. Díaz, E. Cánovas, P. G. Linares, and A. Luque, Phys. Rev. Lett. 97, 247701 (2006). ${ }^{4}$ W. Wang, A. S. Lin, and J. D. Phillips, Appl. Phys. Lett. 95, 011103 (2009).

${ }^{5}$ V. Popescu, G. Bester, M. C. Hanna, A. G. Norman, and A. Zunger, Phys. Rev. B 78, 205321 (2008).

${ }^{6}$ P. J. Carrington, M. C. Wagener, J. R. Botha, A. M. Sanchez, and A. Krier, Appl. Phys. Lett. 101, 231101 (2012).

${ }^{7}$ K. Tanabe, D. Guimard, D. Bordel, and Y. Arakawa, Appl. Phys. Lett. 100, 193905 (2012).

${ }^{8}$ C. E. Pryor and M.-E. Pistol, Phys. Rev. B 72, 205311 (2005).

${ }^{9}$ M. C. Wagener, P. J. Carrington, J. R. Botha, and A. Krier, Appl. Phys. Lett. 103, 063902 (2013).

${ }^{10}$ N. López, L. A. Reichertz, K. M. Yu, K. Campman, and W. Walukiewicz, Phys. Rev. Lett. 106, 028701 (2011).

${ }^{11}$ C.-P. Tsai, S.-C. Hsu, S.-Y. Lin, C.-W. Chang, L.-W. Tu, K.-C. Chen, T.S. Lay, and C.-C. Lin, Opt. Express 22, A359 (2014).

${ }^{12}$ M. C. Wagener, P. J. Carrington, J. R. Botha, and A. Krier, J. Appl. Phys. 115, 014302 (2014).

${ }^{13}$ S. Sauvage, P. Boucaud, J.-M. Gérard, and V. Thierry-Mieg, Phys. Rev. B 58, 10562 (1998).

${ }^{14}$ P. D. Hodgson, R. J. Young, M. Ahmad Kamarudin, P. J. Carrington, A. Krier, Q. D. Zhuang, E. P. Smakman, P. M. Koenraad, and M. Hayne, J. Appl. Phys. 114, 073519 (2013).

${ }^{15}$ R. Strandberg and T. W. Reenaas, J. Appl. Phys. 105, 124512 (2009). 